\title{
HER2/neu testing for anti-HER2-based therapies in patients with unresectable and/or metastatic gastric cancer
}

\author{
Carlos Gómez-Martin, ${ }^{1}$ Elena Garralda, ${ }^{1}$ M José Echarri, ${ }^{2}$ Anabel Ballesteros, ${ }^{3}$ \\ Alberto Arcediano, ${ }^{4}$ José Luis Rodríguez-Peralto, ${ }^{5}$ Manuel Hidalgo, ${ }^{1}$ \\ Fernando López-Ríos ${ }^{6}$
}

${ }^{1}$ Gastrointestinal Cancer Clinical Research Unit, Spanish National Cancer Research Centre,

Madrid, Spain

${ }^{2}$ Medical Oncology Unit, Hospital Universitario Severo Ochoa, Madrid, Spain

${ }^{3}$ Medical Oncology Unit, Hospital Universitario de la Princesa, Madrid, Spain ${ }^{4}$ Medical Oncology Division, Hospital Universitario de Gualajara, Guadalajara, Spain ${ }^{5}$ Pathology Department, Hospital Universitario 12 de Octubre, Madrid, Spain ${ }^{6}$ Laboratorio de Dianas Terapéuticas, Centro Integral Oncológico 'Clara Campal', Hospital Universitario Madrid Sanchinarro, Universidad San Pablo-CEU, Facultad de Medicina, Madrid, Spain

\section{Correspondence to}

Dr Carlos Gómez-Martin, Gastrointestinal Cancer Clinical Research Unit, Clinical Research Programme, Spanish National Cancer Research Centre (CNIO) Fuenlabrada University Hospital, Camino del Molino 2 .

Fuenlabrada 28942, Madrid, Spain; cgomezm@cnio.es

Accepted 20 March 2012

This paper is freely available online under the BMJ Journals unlocked scheme, see http:// jcp.bmi.com/site/about/ unlocked.xhtml

\section{ABSTRACT}

Aim To study the HER2 gene amplification or overexpression in patients with advanced gastric cancer (GC) and their association with patient characteristics and patient survival.

Patients and methods Tumour tissue samples from 148 patients with advanced GC were studied for HER2 by immunohistochemistry $(\mathrm{IHC})$, fluorescence in situ hybridisation (FISH) and dual colour silver enhanced in situ hybridisation (dc-SISH) methods. Clinicopathological data from all patients were collected. Progression free survival and overall survival were also analysed.

Results Mean age was 67 (33-83) years; 75\% were male subjects, and $51 \%$ had intestinal histological type. HER2 + rates were 10.1\% (15/148) by IHC, 18.2\% (27) $148)$ by $\mathrm{FISH}+$ or $21.6 \%(32 / 148)$ by dc-SISH+. There were significant differences in HER2 + rates according to histological type when FISH (intestinal, 23\%; no intestinal, 4\%; $p<0.0001$ ) or dc-SISH (intestinal, 26\%; no intestinal, $6 \% ; p<0.0001)$ amplification techniques were used. Median overall survival was significantly longer in HER2 + patients despite the determination technique used: IHC (21.4 vs 9.8 months, HR 0.42; $p=0.005$ ); FISH (19.6 vs 9.7 months, HR 0.49; $p=0.007$ ) or dc-SISH (19.6 vs 9.7 months, HR 0.53; $p=0.009$ ). Factors associated with favourable survival in the multivariate analysis were intestinal type and Her2+ determination by IHC, FISH or dc-SISH.

Conclusion HER2 gene amplification is significantly associated with patient survival. HER2 gene amplification approaches might be an optimal HER2/neu testing strategy for the selection of HER2 + GC patients who are candidates to be treated with anti-HER2 therapies.

The prognostic value of HER2 in gastric cancer (GC) is controversial and historically its overexpression and/or amplification have been considered a poor prognosis factor. Nevertheless, recent studies appear to cast doubt on this negative view. A randomised phase III trial (ToGA) in advanced GC patients with 3+ HER2 (Human Epidermal growth factor Receptor type 2) overexpression measured by immunohistochemistry (IHC) or with HER2 amplification determined by fluorescence in situ hybridisation (FISH) has demonstrated that trastuzumab added to standard chemotherapy leads to a significant improvement in patients' survival compared with chemotherapy alone, setting a new standard of treatment for these patients. The results of this trial have prompted the use of IHC test followed by FISH in IHC2+ cases for HER2 diagnostics. Dual colour silver enhanced in situ hybridisation (dc-SISH) is a new technique which combines the accuracy of the FISH technique and the morphological control of the IHC technique. In opposition to what has been stated by European Medicines Agency based on the ToGA subanalysis, in this paper we have shown how dc-SISH might be a better approach for the selection of HER2+ GC patients who are candidates to be treated with anti-HER2 therapies.

\section{INTRODUCTION}

GC is one of the most frequent tumours in the world with approximately 989000 new cases and 738000 deaths per year. ${ }^{1}$ In Spain, this tumour is currently the fifth leading cause of cancer-related death in both sexes. ${ }^{2}$ GC is often diagnosed at an advanced stage where curative radical gastrectomy is generally unfeasible and, consequently, survival rate for GC at 5 years of diagnosis is under $27 \%{ }^{3}$ Chemotherapy improves median overall survival (OS) for patients with unresectable and/or metastatic GC; however, no chemotherapy combination has been accepted as the gold standard.

Recently, a randomised phase III Trial in advanced GC patients (ToGA) with 3+ HER2 overexpression measured by IHC or with HER2 amplification determined by FISH has demonstrated that trastuzumab added to standard chemotherapy leads to a significant improvement in OS compared with chemotherapy alone, setting a new standard of treatment for these patients. ${ }^{4}$

The prognostic value of the HER2 protein expression in GC is a controversial topic. ${ }^{5-7}$ Although in most studies HER2 overexpression and/or amplification has been considered a poor prognosis factor, 89 recent studies appear to cast doubt on this negative view particularly after the results of the ToGA (Trastuzumab for Gastric Cancer) study. ${ }^{10} 11$ It remains to be explained whether the longer survival rate achieved with standard chemotherapy in patients with HER2 overexpression and/or amplification is related to the presence of this molecular alteration by itself, the greater percentage of intestinal type cases among these patients or if it is due to factors as yet unknown.

In addition, the results of the ToGA study had prompted the use of IHC test followed by FISH in IHC2 + cases for HER2 diagnostics. Despite FISH being considered the gold standard for HER2 gene 
amplification, a number of barriers such as the need of specialised equipment or the lack of morphological examination control prevent its wider use in the clinical setting. dc-SISH is a new technique which combines the accuracy of the FISH technique and the tissue histopathological features (morphological control) of the IHC technique. ${ }^{12}$ Our group has demonstrated the concordance between the FISH and dc-SISH techniques for determining HER2 gene amplification in GC samples. ${ }^{13}$

The aims of this study were to determine the frequency of HER2 gene amplification by FISH and dc-SISH techniques and overexpression by IHC in patients with advanced GC, and to evaluate the association of HER2 gene amplification or overexpression with patient's characteristics and survival, particularly in the subgroup of intestinal type cases.

\section{MATERIALS AND METHODS Patients' eligibility}

Tumour samples and medical records from patients who had been consecutively evaluated in our institutions for advanced GC treatment between January 2007 and December 2009 were retrospectively reviewed.

Eligibility criteria included patients with histologically confirmed advanced or/metastatic GC who had measurable and no measurable lesions by CT scan according to the Response Evaluation Criteria in Solid Tumours v1.0 ${ }^{14}$ who had not received previous treatment at the time of diagnosis. Patients had received treatment in line with the institution usual practice and data on tumour response had been assessed according to Response Evaluation Criteria in Solid Tumours criteria and recorded in the patient's medical record. Tumour samples were obtained prior to any chemotherapy or systemic treatment.

The study was performed after approval by the Local Research Ethics Committee and in accordance with the Declaration of Helsinki, the Good Clinical Practices, and local ethical and legal requirements.

\section{Study assessments}

Clinical data were collected from medical records by a single clinician blinded to HER2 status, using a datasheet specifically developed for this study. Clinicopathological data such as gender, age, histological type according to the Lauren's classification $^{15}$ number and locations of metastases, previous surgery for GC and chemotherapy treatment received were reviewed.

Progression free survival (PFS), OS and clinical benefit rate (patients with either long lasting stabilisation of disease or objective partial or total response) were assessed from individual patient's medical records. OS was measured from the date of diagnosis of metastatic disease until the date of death or last follow-up if alive. PFS was defined as the time from date of diagnosis of metastatic disease until first documentation of PD or death for any cause.

All $H \& E$ slides and paraffin blocks were centralised in a referral laboratory (Laboratorio de Dianas Terapeuticas). Two of the investigators (CGM, FLR) selected the most appropriate block and performed the interpretation of the different assays in a blinded manner to each other's results. Also, $4 \mu \mathrm{m}$-thick sections were cut and placed on charged polylysine-coated slides.

To determine the frequency of HER2 gene amplification or overexpression in the tumour tissue samples the following assay methods were used: IHC, FISH and dc-SISH. The real-time PCR (RT-PCR) technique was used to study discordant cases. HER2+ disease $(\mathrm{HER} 2+)$ was defined as IHC3 + or FISH+ (FISH ratio $\geq 2$ ) or dc-SISH+ (dc-SISH ratio $\geq 2$ ).

Due to the retrospective nature of this study, none of the obtained results was used to modify the treatment of patients.
Table 1 Patient characteristics

\begin{tabular}{|c|c|}
\hline Patient characteristics & $N=148$ \\
\hline \multicolumn{2}{|l|}{ Age (years) } \\
\hline Mean $\pm S D$ & $67 \pm 9.6$ \\
\hline Range (min $-\max$ ) & $33-83$ \\
\hline \multicolumn{2}{|l|}{ Sex, n (\%) } \\
\hline Male & $111(75)$ \\
\hline Female & $37(25)$ \\
\hline \multicolumn{2}{|l|}{ Tumour sample origin, $\mathrm{n}(\%)$} \\
\hline $\begin{array}{l}\text { Endoscopic biopsy from primary } \\
\text { tumour }\end{array}$ & $40(27.0)$ \\
\hline Surgical specimen from primary tumour & $96(64.8)$ \\
\hline Surgical specimen from metastases & $12(8.1)$ \\
\hline \multicolumn{2}{|l|}{ First diagnosed with metastases, n (\%) } \\
\hline Yes & $86(58.11)$ \\
\hline No & $62(41.89)$ \\
\hline \multicolumn{2}{|l|}{ Lauren's histological type, n (\%) } \\
\hline Intestinal & $75(50.7)$ \\
\hline Diffuse & $42(28.3)$ \\
\hline Undetermined & $31(20.9)$ \\
\hline \multicolumn{2}{|l|}{ Localisation of the metastases* ${ }^{*}, \mathrm{n}(\%)$} \\
\hline Liver & $57(38.5)$ \\
\hline Lung & $17(11.5)$ \\
\hline Thoracic lymph nodes & $15(10.1)$ \\
\hline Abdominal lymph nodes & $90(60.8)$ \\
\hline Peritoneal spread & $48(32.4)$ \\
\hline $\begin{array}{l}\text { Other (bone, ovarian, central nervous } \\
\text { system) }\end{array}$ & $14(9.5)$ \\
\hline \multicolumn{2}{|l|}{ Treatment, n (\%) } \\
\hline Chemotherapy & $121(81.8)$ \\
\hline Monotherapy & $11(7.4)$ \\
\hline Platinum/fluoropyrimidines doublet & $34(23.0)$ \\
\hline Platinum/taxane doublet & $29(19.6)$ \\
\hline Other doublets & $2(1.4)$ \\
\hline Platinum-based triplet & $25(16.9)$ \\
\hline Trastuzumab + chemotherapy & $20(13.5)$ \\
\hline Surgery & $2(1.4 \%)$ \\
\hline None & $25(16.9)$ \\
\hline
\end{tabular}

\section{Immunohistochemistry}

The HER2 protein status was determined by IHC using the Pathway anti-HER2/neu (4B5) antibody in the fully automated platform BenchMark ULTRA®) (Ventana Medical Systems, Inc, Tucson, Arizona, USA). The slides were examined and scored independently by two experienced pathologists according to a previously defined score proposed by Ruschoff et al. ${ }^{16}$

\section{Fluorescence in situ hybridisation}

HER2 copy number was investigated by FISH using the PathVysion HER2 DNA probe kit (Vysis Inc, Abbot Laboratories, Illinois, USA) with the DAKO Histology FISH Accessory kit. The manufacturer's instructions were modified using DAKO Histology kit in order to optimise the technique. ${ }^{17}$

Dual colour silver enhanced in situ hybridisation

Automated dc-SISH was performed on Ventana Benchmark XT (Ventana Medical Systems). INFORM HER2 DNA Probe and INFORM Chromosome 17 Probe were visualised on the same slide following manufacturer's protocols with a few variations. Assay conditions were modified for optimal results. The entire assay procedure (deparaffinisation, pretreatment, hybridisation, stringency wash, signal detection and counterstaining) was fully automated. ${ }^{18}$ 
Table 2 Clinicopathological correlation data

\begin{tabular}{|c|c|c|c|c|c|c|c|}
\hline & & IHC3+ & $\mathrm{IHC} \leq 2$ & $\begin{array}{l}\text { FISH } \\
\text { ratio } \geq 2\end{array}$ & $\begin{array}{l}\text { FISH } \\
\text { ratio <2 }\end{array}$ & $\begin{array}{l}\text { dc-SISH } \\
\text { ratio } \geq 2\end{array}$ & $\begin{array}{l}\text { dc-SISH } \\
\text { ratio }<2\end{array}$ \\
\hline \multicolumn{8}{|c|}{ Histology type (Lauren classification), $\mathrm{n}$} \\
\hline Intestinal (\%) & $75(50.7)$ & 11 & 64 & 23 & 52 & 26 & 49 \\
\hline No intestinal (\%) & $73(49.3)$ & 4 & 69 & 4 & 69 & 6 & 67 \\
\hline$p$ Value* & & 0.0641 & & $<0.0001$ & & $<0.0001$ & \\
\hline \multicolumn{8}{|c|}{ Site of metastases, $n$} \\
\hline Liver & & 7 & 50 & 14 & 43 & 17 & 40 \\
\hline p Value* & & 0.2694 & & 0.1152 & & 0.0550 & \\
\hline Lung & & 4 & 13 & 5 & 12 & 7 & 10 \\
\hline p Value* & & 0.1848 & & 0.1987 & & 0.0564 & \\
\hline Bone & & 0 & 8 & 0 & 8 & 0 & 8 \\
\hline p Value* & & 0.6171 & & 0.3516 & & 0.2018 & \\
\hline Peritoneal & & 3 & 45 & 5 & 43 & 5 & 43 \\
\hline p Value* & & 0.0604 & & 0.0876 & & 0.0218 & \\
\hline
\end{tabular}

Resolution of discordant cases: RT-PCR

Tumours with discordant results obtained by FISH and dc-SISH and those with negative $(0 / 1+)$ IHC and amplified by FISH or dc-SISH were analysed by RT-PCR using the LightMix kit HER2/neu (Roche Diagnostics, Penzberg, Germany). A 101 bp fragment of the HER2 gene and a $119 \mathrm{bp}$ fragment of the RPL23 reference gene, both localised on chromosome 17q21, were amplified according to the manufacturer's instructions.

A complete description of IHC, FISH, dc-SISH and RT-PCR, as well as procedures of the postanalytical phase, has been described elsewhere. ${ }^{13}$

\section{Statistical analysis}

The primary objective was to determine the frequency of HER2 gene amplification or overexpression in patients with advanced GC. Comparisons of the frequency of HER2 overexpression or amplification for different groups were performed using the Wilcoxon-Mann-Whitney test (for two groups) or the Krus$\mathrm{kal}-$ Wallis test (for more than two groups).

Secondary objectives were to evaluate the association of HER2 gene amplification or overexpression with patient's characteristics and patient survival. For categorical variables, the possible association between the result of the technique used (IHC, FISH, dc-SISH) and the clinicopathological parameters was analysed using the Fisher's exact test, $\chi^{2}$ test or Cochran-Mantel-Haenszel test (to compare one, two or more than two groups, respectively). Survival analyses (OS and PFS) according to HER2+ status were performed using the Kaplan-Meier method and differences between the patient groups were tested by the log-rank test. OS analyses were also performed according to the presence of intestinal versus no intestinal disease.
Multivariate survival analyses were determined using a Cox proportional hazards regression model for each of the HER2 variables together with clinical variables recognised as prognostic factors (peritoneal spread, non-intestinal type and presence of hepatic metastases) including risk ratios and 95\% CI. A subgroup analysis based on tumour histology was also done. All tests were two sided, and $p$ values $<0.05$ were considered statistically significant. All statistical analyses were performed using SPSS V.15.0 for Windows (IBM SPSS Inc).

\section{RESULTS}

\section{Patients' characteristics}

Complete clinicopathological information was obtained from medical records in 148 out of the 166 patients reviewed. All tumour samples were evaluated with all assays. Most patients $(75 \%, \mathrm{n}=111)$ were male subjects with a mean age of $67 \pm 9.6$ years, ranging from 33 to 83 years.

Histological examinations from biopsies were obtained during an endoscopy in $40(27.0 \%)$ patients and from surgical specimens in $108(72.9 \%)$ patients of whom $12(8.1 \%)$ were from distant metastases (eight peritoneal implants and four liver metastases). Histopathological diagnosis was intestinal type in $75(50.7 \%)$ patients, diffuse type in $42(28.3 \%)$ patients and undetermined type in $31(20.9 \%)$ patients.

Most patients $(81.8 \%, \mathrm{n}=121)$ received chemotherapy, which was platinum-based in $42.6 \%(n=63)$. In $13.5 \%(n=20)$, trastuzumab was added to the chemotherapeutic regimen. Clinicopathological data are detailed in table 1.

\section{Frequency of HER2 gene amplification or overexpression}

Results from the HER2 overexpression by IHC analysis resulted in the following classification: 122 patients with score 0 (82.4\%),

Table 3 Progression free survival and overall survival according to HER2 overexpression/HER2 amplification results

\begin{tabular}{|c|c|c|c|c|c|c|}
\hline & IHC3+ & $\mathrm{IHC} \leq 2$ & FISH ratio $\geq 2$ & FISH ratio $<2$ & dc-SISH ratio $\geq 2$ & dc-SISH ratio $<2$ \\
\hline med PFS (months) & 7.98 & 5.45 & 7.98 & 5.35 & 7.98 & 5.35 \\
\hline HR $(95 \%$ Cl) & \multicolumn{2}{|c|}{$\mathbf{0 . 6 2}(0.34$ to 1.13$)$} & \multicolumn{2}{|c|}{$\mathbf{0 . 5 8}(0.36$ to 0.94$)$} & \multicolumn{2}{|l|}{$\mathbf{0 . 6 1}(0.39$ to 0.95$)$} \\
\hline$p$ Value & \multicolumn{2}{|c|}{0.119} & \multicolumn{2}{|c|}{0.027} & \multicolumn{2}{|l|}{0.029} \\
\hline HR $(95 \% \mathrm{Cl})$ & \multicolumn{2}{|c|}{$\mathbf{0 . 4 2}(0.21$ to 0.84$)$} & \multicolumn{2}{|c|}{$\mathbf{0 . 4 9}(0.29$ to 0.83$)$} & \multicolumn{2}{|l|}{$0.53(0.33$ to 0.85$)$} \\
\hline $\mathrm{p}$ Value & \multicolumn{2}{|l|}{0.005} & \multicolumn{2}{|l|}{0.007} & \multicolumn{2}{|l|}{0.009} \\
\hline
\end{tabular}

dc-SISH, dual colour silver enhanced in situ hybridisation; FISH, fluorescence in situ hybridisation; IHC, immunohistochemistry; med PFS, median progression free survival; med OS, median overall survival. 
Figure 1 Overall survival according to HER2 overexpression by immunohistochemistry (IHC) (A), HER2 gene amplification by fluorescence in situ hybridisation (FISH) (B) or dual colour silver enhanced in situ hybridisation (SISH) (C) techniques.
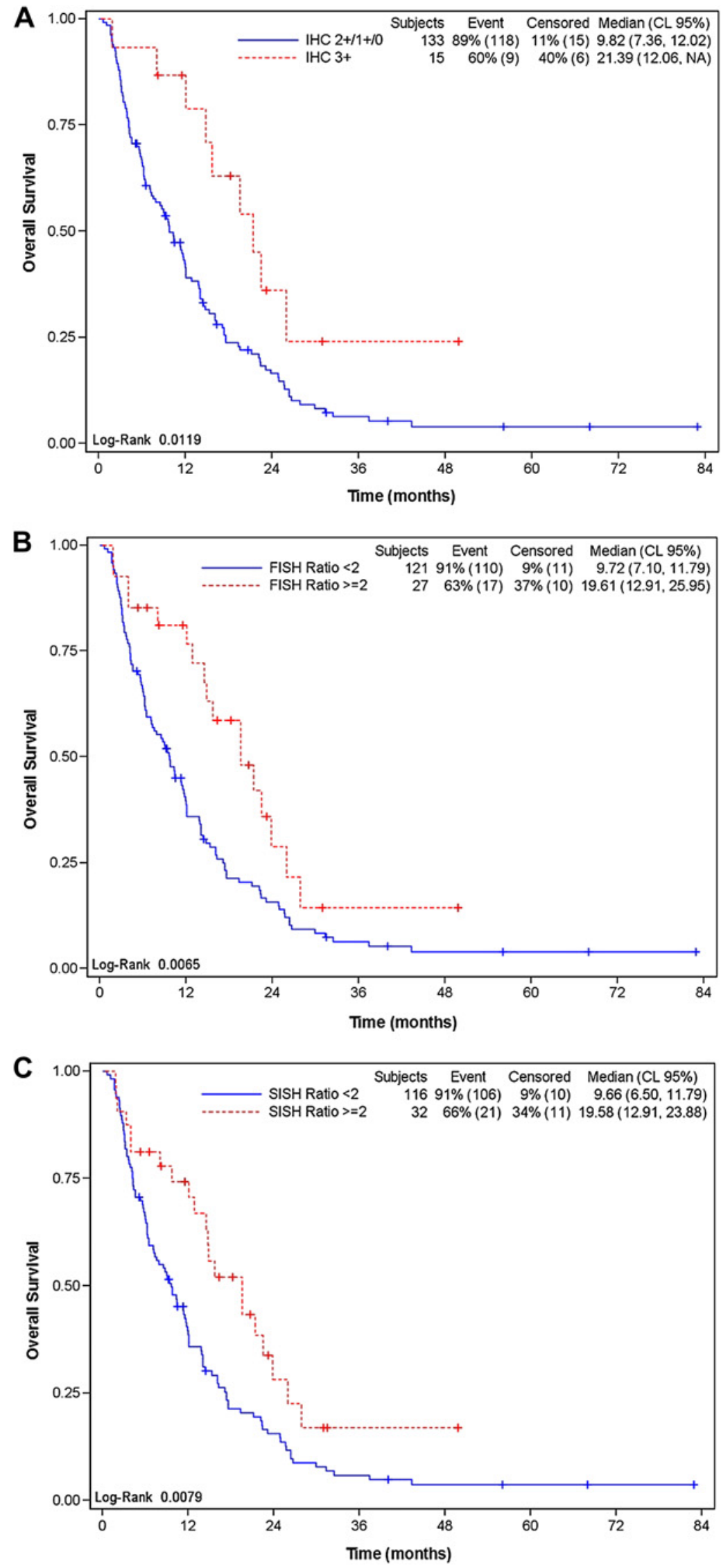

five patients with score $1+(3.4 \%)$, six with score $2+(4.1 \%)$ and 15 with score $3+(10.1 \%)$, with this last category being considered the IHC+ group.
HER2 amplification by FISH obtained 27 cases of HER2 oncogen amplification. Mean ratio of HER2 to CEP17 was 5.81 (95\% CI 4.64 to 6.98 ) and range 2.1-12.3. HER2 amplification 
by dc-SISH was observed in 32 cases, with a mean ratio of 6.87 (95\% CI 5.51 to 8.23 ) and range 2.1-16.7.

\section{Clinicopathological correlation with HER2 gene amplification or overexpression}

There were no statistically significant differences in HER2 overexpression by IHC results according to sex, age or metastatic site. Likewise, no statistically significant differences were observed in HER2 amplification results obtained by FISH or dcSISH according to age and sex. However, according to metastatic site a lower incidence of amplified results was obtained for patients with peritoneal carcinomatosis when dc-SISH technique was used, and this difference was statistically significant $(\mathrm{p}=0.0218)$ (table 2).

All the three techniques used were highly positive for the intestinal histological type: $73.3 \%$ of cases with IHC overexpression, $85.1 \%$ cases with FISH amplification and $81.25 \%$ of cases amplified by dc-SISH corresponding to the intestinal type. However, a statistically significant association was found only between the presence of intestinal type and a positive result in the FISH or dc-SISH amplification techniques $(\mathrm{p}<0.0001)$ (table 2).

\section{Discordant cases}

Samples amplified by FISH and dc-SISH, considered as a positive control for HER2 gene amplification, were all found to be amplified using the RT-PCR quantitative approach. Similarly, samples assessed as negative for HER2 gene amplification by both FISH and dc-SISH gave a normalised ratio of HER2 to RPL $23 \sim 1$ (range from 0.836 to 1.0) which is below the cut-off limit of 2 and confirms the absence of amplification. No amplification by FISH was observed in six out of the 34 cases amplified by dc-SISH (cases 8, 39, 67, 68, 113 and 129). The dc-SISH amplification ratio was between 2 and 3 and all of them were IHC 0/1 + . All these cases were polysomic and were confirmed as negative for amplification by RT-PCR.

In five cases, the IHC result was $0 / 1+$ despite amplification either by FISH or by dc-SISH techniques. In three of these five cases there was no remaining tissue to perform an appropriate RT-PCR analysis. For the last two cases the result of RT-PCR confirmed the HER2/neu amplification shown by FISH and dc-SISH tests.

\section{Patient survival correlation with HER2 gene amplification or overexpression}

Median PFS was 6.14 months (95\% CI 5.19 to 7.23 months) and median OS was 11.37 months (95\% CI 9.00 to 13.83 months) for the entire population.

No differences were observed in PFS according to HER2 overexpression, although a statistically significant difference was observed when groups were analysed according to the presence or amplification assessed by FISH or dc-SISH (table 3).

Mean OS was significantly longer in the group of patients with HER2 overexpression or amplification, whatever the technique used for its determination: IHC (21.4 vs 9.8 months, HR 0.42; $\mathrm{p}=0.005$ ), FISH (19.6 vs 9.7 months, HR 0.49; $\mathrm{p}=0.007)$ or dc-SISH (19.6 vs 9.7 months, HR $0.53 ; \mathrm{p}=0.009$ ) (table 3; figure 1).

Median OS for the subgroup of patients with intestinal type GC (14.5 months; $95 \%$ CI 12.02 to 21.16$)$ was significantly higher to that observed in the no intestinal group (7.36 months, ( $95 \%$ CI 6.24 to 10.31) (log-rank $\mathrm{p}=0.0011$ ).

\section{Multivariate survival analyses}

For the entire patient population, the presence of peritoneal carcinomatosis was found to be a negative prognostic factor for OS (HR: $1.60 ; 95 \%$ CI 1.08 to 2.38; $\mathrm{p}=0.020$ ), while the presence of intestinal histological type (intestinal vs no intestinal: HR: 0.68 ; $95 \%$ CI 0.46 to 0.99 ; $\mathrm{p}=0.044)$, the HER 2 protein overexpression assessed by IHC (IHC3+ vs IHC $\leq 2$ : HR: 0.47; $95 \%$ CI 0.24 to $0.94 ; p=0.032$ ), or the HER2 gene amplification by FISH (ratio $\geq 2$ vs ratio <2: HR: 0.58 ; $95 \%$ CI 0.34 to 0.99 ; $\mathrm{p}=0.046$ ) or by dc-SISH (ratio $\geq 2$ vs ratio $<2$ : HR: $0.58 ; 95 \%$ CI 0.36 to $0.93 ; \mathrm{p}=0.023$ ) was found to be a favourable prognostic factor.

The same analysis was performed for the subgroup of patients with intestinal type. In this case, no prognostic value for HER2 protein overexpression or for its amplification determined by FISH was observed. Only the presence of hepatic metastases
Figure 2 Overall survival according to Lauren's histological type of carcinoma with or without HER2 gene amplification by dual colour silver enhanced in situ hybridisation.

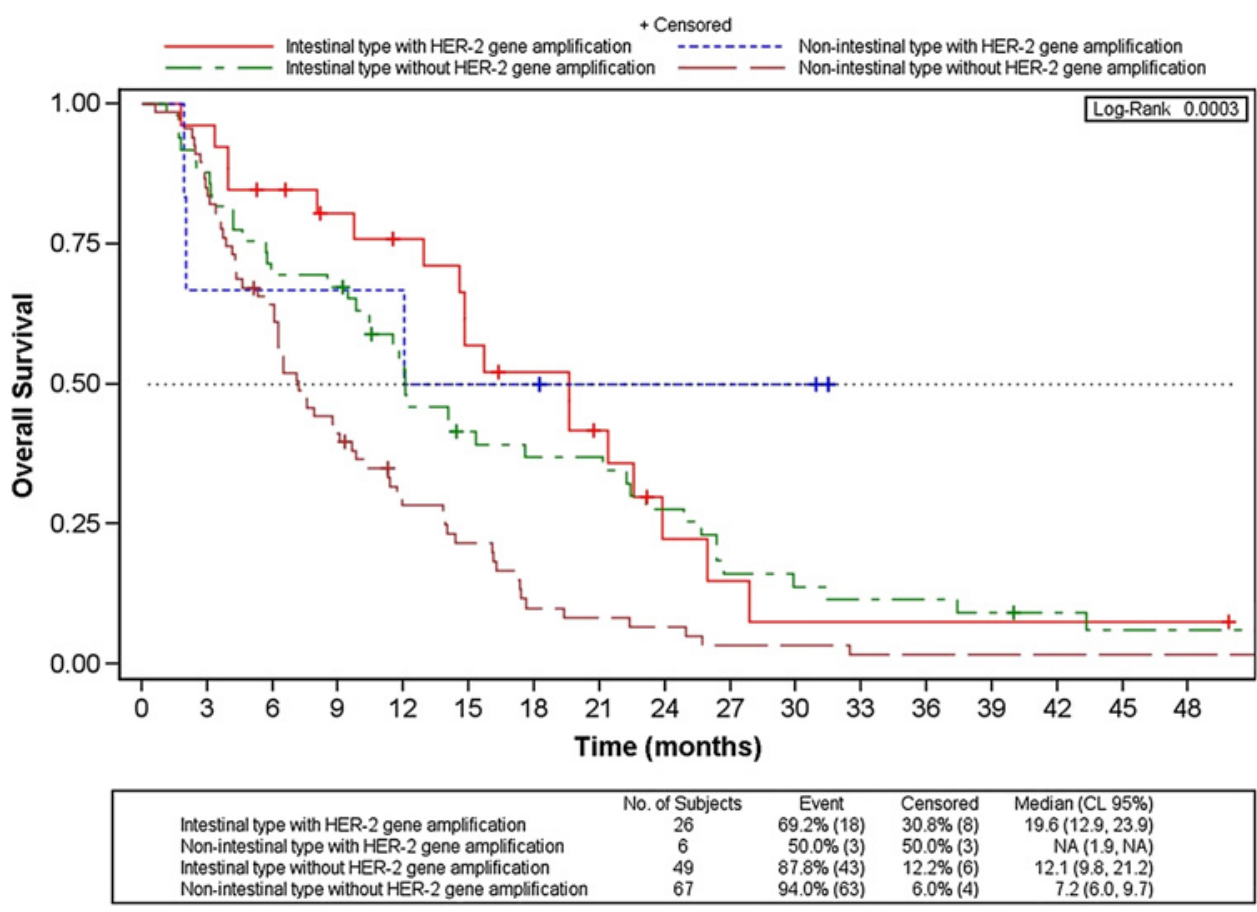


(HR: $1.74 ; 95 \%$ CI 1.03 to $2.94 ; \mathrm{p}=0.038$ ) or peritoneal carcinomatosis (HR: 2.87; 95\% CI 1.56 to 5.30; $\mathrm{p}=0.0007$ ) was statistically significant.

When OS was analysed using a Kaplan-Meier model and stratifying patients according to both their Lauren's histological type and positive or negative result of IHC or ISH tests, only dcSISH was able to find significant differences between the four subgroups of patients ( $p=0.0003$, log-rank test). The Cox regression analysis model showed similar results $(p=0.0005)$ (figure 2).

\section{DISCUSSION}

Results of the clinicopathological correlation with HER2 status in our series are similar to those described in previous studies, with a high correlation with the presence of the intestinal histological type, particularly when HER2 amplification techniques were used. ${ }^{19} 20$

On univariate analysis, median OS was significantly longer in HER2 + patients, despite the determination technique used, and this prognostic value was confirmed in the multivariate survival analyses when dc-SISH was used. These findings corroborate phase III ToGA study ${ }^{4}$ results, wherein the significant improvement in the mean OS observed with the addition of trastuzumab to chemotherapy in patients with HER2+ metastatic GC was mainly the result of the survival advantage conferred to patients with high expression of the HER2 protein and/or confirmed HER2 amplification by FISH. Noteworthy, in the three HER2 determination techniques used the mean OS rates in HER2 + patients in our study almost doubled those achieved in HER2 patients.

For the whole population, HER2-positivity and the intestinal type were found to be factors associated with a more favourable survival in advanced GC patients in the multivariate survival analyses. The same conclusion was reached in a US and European collaborative analysis recently presented by Shah et al at the ASCO 2011 meeting. ${ }^{10}$ However, in the subgroup analysis based on tumour histology and HER2 status, only HER2 amplification determined by means of dc-SISH was deemed to be of prognostic value for intestinal type patients.

In addition, the presence of peritoneal carcinomatosis was found to be a negative prognostic factor for OS in the whole group of patients or when analysing only the subgroup of intestinal patients.

Despite not being a randomised study (which can be considered a potential weakness of our trial) patients were not selected but were included consecutively as soon as they were referred for assessment; this fact diminishes the possible selection bias.

The three diagnostic techniques used (IHC, FISH and dcSISH) have demonstrated to be comparable for HER2 status assessment. ${ }^{13}$ Only a low percentage of discordant cases were observed between FISH and dc-SISH or between IHC and FISH, which were resolved by quantitative RT-PCR.

Even if the three techniques were similar for assessing the subgroup of patients with favourable prognosis, only $10 \%$ of HER 2 + cases were detected by IHC compared with 18\%-22\% of HER $2+$ cases detected by hybridisation techniques. In addition, there is a minor subjectivity in the interpretation of results with the hybridisation techniques compared with the IHC method, for which three different criteria have been developed in the last 3 years. ${ }^{15} 2122$ Hybridisation techniques are less timedepending (signal permanency) and easier to be reproduced interlaboratory and intralaboratory, particularly with the brightfield microscopic technique adopted in the dc-SISH method, which have been validated by our group. ${ }^{13}$

\section{Take-home messages}

HER2 gene amplification studied by either fluorescence in situ hybridisation or dual colour silver enhanced in situ hybridisation is significantly associated with favourable survival.

- HER2 gene amplification approaches might be an optimal testing strategy for the selection of patients who are candidates to be treated with anti-HER2 therapies.

In view of all these advantages, and in opposition to what has been stated by European Medicines Agency based on the ToGA subanalysis, we consider that the hybridisation techniques in general, and dc-SISH in particular, might be a better approach for the selection of HER2 + GC patients who are candidates to be treated with anti-HER2 therapies. Interestingly, a similar conclusion has been reached in breast carcinomas and proposed in gastric carcinoma, given the inconsistencies in HER2 IHC testing. ${ }^{2-24}$ In this regard, a comprehensive analysis of some of the ToGA subgroups would be most valuable in providing robust guidelines for HER2 testing.

Acknowledgements We are most grateful to the staff of the Laboratorio de Dianas Terapéuticas for their help throughout this study.

Funding Financial support of research provided by Roche Farma Spain who have no role in conception and design of this research, analysis and interpretation of data, drafting of the article or final approval of the version to be published.

\section{Competing interests None.}

Patient consent We report our results in a series of gastric cancer patients treated for their disease. There are no personal data in our manuscript. All patients who are alive were provided with an Informed consent document to allow the researchers perform this study by using their tumoral samples.

Ethics approval The ethics approval was provided by the Ethics Committee/ Institutional Review Board at Centro Integral de Oncologia Clara Campal.

Provenance and peer review Not commissioned; externally peer reviewed.

\section{REFERENCES}

1. Ferlay J, Shin HR, Bray F, et al. Estimates of worldwide burden of cancer in 2008 : GLOBOCAN 2008. Int J Cancer 2010;127:2893-917.

2. Cabanes A, Perez-Gomez B, Aragones N, et al. La situación del cáncer en España, 1975-2006. Spain: INE- Madrid, 2009.

3. Howlader N, Noone AM, Krapcho M, et al. SEER Cancer Statistics Review, 1975 2008. National cancer Institute Bethesda, http://seer.cancer.gov/csr/1975_2008/, based on November 2010 SEER data submission, posted to the SEER web site, 2011 U.S.National Institutes of Helath, USA

4. Bang YJ, Van Cutsem E, Feyereislova A, et al; GA Trial Investigators. Trastuzumab in combination with chemotherapy versus chemotherapy alone for treatment of HER2 positive advanced gastric or gastro-oesophageal junction cancer (ToGA): a phase 3 open-label, randomised controlled trial. Lancet 2010;376:687-97.

5. Grabsch H, Sivakumar S, Gray S, et al. HER2 expression in gastric cancer: rare, heterogeneous and of no prognostic value-conclusions from 924 cases of two independent series. Cell Oncol 2010;32:57-65.

6. Gurel S, Dolar E, Yerci O, et al. The relationship between c-erbB-2 oncogene expression and clinicopathological factors in gastric cancer. J Int Med Res 1999;27:74-8.

7. Park DI, Yun JW, Park JH, et al. HER-2/neu amplification is an independent prognostic factor in gastric cancer. Dig Dis Sci 2006;51:1371-9.

8. Tanner M, Hollmen M, Junttila TT, et al. Amplification of HER-2 in gastric carcinoma: association with Topoisomerase llalpha gene amplification, intestinal type, poor prognosis and sensitivity to trastuzumab. Ann Oncol 2005;16:273-8.

9. Yan B, Yau EX, Bte Omar SS, et al. A study of HER2 gene amplification and protein expression in gastric cancer. J Clin Pathol 2010;63:839-42.

10. Shah MA, Janjigian YY, Pauligk C. Prognostic significance of human epidermal growth factor-2 (HER2) in advanced gastric cancer: a U.S. and European international collaborative analysis. J Clin Oncol 2011;29(suppl; abstr 4014).

11. Yoon HH, Shi Q, Sukov WR. HER2 expression/amplification: frequency, clinicopathologic features, and prognosis in 713 patients with esophageal adenocarcinoma (EAC). J Clin Oncol 2011;29(suppl; abstr 4012).

12. Penault-Llorca F, Bilous $M$, Dowsett $M$, et al. Emerging technologies for assessing HER2 amplification. Am J Clin Pathol 2009;132:539-48. 
13. García-García E, Gómez-Martín C, Angulo B, et al. Hybridization for HER2 testing in gastric carcinoma: a comparison of fluorescence in situ hybridization with a novel fully automated dual-color silver in situ hybridization. Histopathology 2011:59:8-17.

14. Therasse $\mathbf{P}$, Arbuck SG, Eisenhauer EA, et al. New guidelines to evaluate the response to treatment in solid tumors. European Organization for Research and treatment of cancer, National cancer Institute of the United States, National cancer Institute of Canada. J Natl Cancer Inst 2000;92:205-16.

15. Lauren P. The two histological main types of gastric carcinoma: diffuse and socalled intestinal-type carcinoma. An attempt at a histo-clinical classification. Acta Pathol Microbiol Scand 1965;64:31-49.

16. Ruschoff J, Dietel M, Baretton G, et al. HER2 diagnostics in gastric cancer-guideline validation and development of standardized immunohistochemical testing. Virchows Arch 2010;457:299-307.

17. Tubbs RR, Hicks DG, Cook J, et al. Fluorescence in situ hybridization (FISH) as primary methodology for the assessment of HER2 Status in adenocarcinoma of the breast: a single institution experience. Diagn Mol Pathol 2007;16:207-10.

18. Nitta $\mathbf{H}$, Hauss-Wegrzyniak B, Lehrkamp $M$, et al. Development of automated brightfield double in situ hybridization (BDISH) application for HER2 gene and chromosome 17 centromere (CEN 17) for breast carcinomas and an assay performance comparison to manual dual color HER2 fluorescence in situ hybridization (FISH). Diagn Pathol 2008;3:41

19. Barros-Silva JD, Leitao D, Afonso L, et al. Association of ERBB2 gene status with histopathological parameters and disease-specific survival in gastric carcinoma patients. Br J Cancer 2009;100:487-93.

20. Yan SY, Hu Y, Fan JG, et al. Clinicopathologic significance of HER-2/neu protein expression and gene amplification in gastric carcinoma. World J Gastroenterol 2011:17:1501-6.

21. Hofmann M, Stoss 0 , Shi D, et al. Assessment of a HER2 scoring system for gastric cancer: results from a validation study. Histopathology 2008;52:797-805.

22. Sauter G, Lee J, Bartlett JM, et al. Guidelines for human epidermal growth factor receptor 2 testing: biologic and methodologic considerations. J Clin Oncol 2009;27:1323-33.

23. Salto-Tellez M, Yau EX, Yan B, et al. Where and by whom should gastric cancer HER2/neu status be assessed?: lessons from breast cancer. Arch Pathol Lab Med 2011;135:693-5.

24. Yan B, Yau EX, Choo SN, et al. Dual-colour HER2/Chromosome 17 chromogenic in situ hybridisation assay enables accurate assessment of HER2 genomic status in gastric cancer and has potential utility in HER2 testing of biopsy samples. J Clin Pathol 2011:64:880-3. 